the species. So different are they in fact that they approach the limits of intrageneric relationship when compared with other fish taxa. This recent article by Pantelouris et al. and the substantial body of literature on eels that has accumulated since 1959 all tend to support Schmidt's classical hypothesis.

\section{ULTRASONICS \\ Recent Applicutions}

trom a Correspondent

THE "Ultrasonics 1971" conference and exhibition was held in London on September 28 and 29 and was the seventh of the series organized by the journal Ultrasonics.

On the opening morning, the session on underwater sound was notable for an account by Professor W. D. Chesterman and Dr J. C. Hopkins (Bath University of Technology) of the recording of side-scan sonar signals on magnetic tape-to be replayed later under controlled conditions or into an oscillograph system so that the pictures can be filmed to give true-to-scale survey records. In a fascinating contribution, Dr R. W. G. Haslett (Kelvin Hughes Ltd, Ilford) described the use of sound in fishing and indicated how model investigations on the echoes could be correlated with the average size and shape of fish in a shoal.

Drs M. Topp and P. Eisenklam (Imperial College, London) drew attention to the importance of ultrasonic atomizers in oil burners, as medical nebulizers, as metal sprayers and the like; their use in Britain is, however, not extensive. Ultrasonic atomizers derive their power from magnetostrictive transducers (20 to 125 $\mathrm{kHz})$ or piezoelectric transducers $(30$ $\mathrm{kHz}$ to $5 \mathrm{MHz}$ ). One such ultrasonic atomizer involves an angular bowlshaped concentrator which is capable of atomizing a $100 \mu \mathrm{m}$ sheet of solid aluminium placed across the mouth, using only a $100 \mathrm{~W}$ generator. Although ultrasonic cavitation produces much more drastic effects in polymer solutions than other degradation techniques, Dr P. Vaughan (Letchworth College, Hertfordshire) emphasized that such processing by ultrasound still remains a research undertaking. In the biological field, Drs W. I. Coakley and C. J. James (University College, Cardiff) spoke on the activity of enzymes released from yeast by sonification. They showed that the rate constant for enzymatic denaturation is dependent on the protein concentration and that for optimum results the cell concentration should be high.

In the afternoon session certain general aspects of ultrasonic testing were considered and there was one very significant contribution by Drs E. E.
Aldridge and H. G. Tattersall (NDT Centre, AERE, Harwell, who have developed the idea that use could be made of the ultrasound radiation scattered from the main wave. Although in general this radiation will not give an absolute measure of the macrostructure of the medium, it can yield useful results when correlated with other relevant information.

High power ultrasonics has come into prominence again and was the theme of one of the parallel morning sessions of the second day; two of the subjects dealt with concerned transducers (Dr R. R. Whymark, Interand Corporation, Chicago, and Dr E. A. Neppiras, University of Houston). Dr B. Largenecker (PVC Laboratory, Waldbach, Austria) showed how the application of high amplitude ultrasound reduces the external stress necessary for plastic deformation; he has made a notable contribution to the design of machines for drawing brittle materials. The second of the morning sessions was devoted to the fast growing field of medical ultra- sonics. Outstanding in this section was the talk by Dr M. K. Patrick (Birmingham General Hospital) on her experience with ultrasonic therapy in clinical practice.

The final session in the afternoon of September 29 was concerned with automated non-destructive testing and the last contributions were from Drs V. M. Barborovsky and D. Marsh (Tube Investments Research Laboratories, Cambridge) on a Schlieren study of ultrasonic pulse propagation and reflexion. The aim of the work was to simulate the detection of cracks in steel tubes and thus to improve the reliability of the test method. Glass models were used in the Schlieren system to provide a transparent medium, ultrasonically very similar to steel. The pulsed light source has to satisfy the condition that its flash duration time was short compared with the period of the ultrasonic wave and a new version of the argon jet source was found to be very suitable. The apparatus had to be carefully designed for vibration isolation because the deflected light

\title{
Recognition Sites for tRNA Charging Enzymes
}

IN Nature New Biology next Wednesday, Squires and Carbon report a series of experiments which shed new light on the intriguing question of how the different species of transfer RNAs are recognized and distinguished by their specific amino-acyl tRNA synthetases, the enzymes which charge tRNA molecules with amino-acids. And into the bargain Squires and Carbon report the location on the Escherichia coli genetic map of the third species of glycine tRNA, tRNA ${ }_{B}^{\mathrm{Gly}}$, which translates the glycine codons GGU/C.

Squires and Carbon found that a mutant strain of $E$. coli which lacks about one-third of the amount of tRNA ${ }_{3}^{\mathrm{Gly}}$ present in wild type cells contains a new species of glycine transfer RNA, designated tRNA $A_{\text {ins }}^{\text {Gly }}$, which can translate the codons GGA/G. This mutated form of glycine transfer RNA maps at about 86 minutes on the circular $E$. coli genetic map and experiments with diploid and merodiploid cells prove that this is the position of the structural gene, or rather genes for tRNA ${ }_{3}^{\mathrm{Gly}}$, of which there must be multiple copies in each wild type genome.

To prove that $\mathrm{RNA}_{\text {ins }}^{\mathrm{Gly}}$ appears as a result of a mutation in one of the tRNA ${ }_{3}{ }_{3}$ structural genes, Squires and Carbon analysed the base sequences of the two molecules and found that they differ in only one position; the anticodon sequence of tRNA ${ }_{3}^{\mathrm{Gly}}$ is GCC and that of tRNA $A_{i n s}^{\text {Gly }}$ is UCC, but apart from this $\mathrm{G} \rightarrow \mathrm{U}$ base change in the third or "wobble" position of the anticodon the two molecules are identical.

Glycyl tRNA synthetase charges the mutated tRNA $\mathrm{Ans}_{\mathrm{lng}}^{\text {oly }}$ at about the same rate as tRNA ${ }_{3}^{\mathrm{Aly}}$. By contrast, Carbon and his colleagues, working with wild type and mutated forms of the two other species of glycine tRNAs (tRNA ${ }_{1}^{\mathrm{aly}}$ and tRNA ${ }_{2}^{\text {aly }}$ ) in $E$. coli, have found that changes in either the first or second base of the anticodon drastically impair the recognition of these molecules by the glycine charging enzyme. They conclude therefore that the first two bases but not the third "wobble" base are involved in the interaction between glycyl tRNA synthetase and the glycine tRNAs.

Squires and Carbon also noticed that the base sequence of tRNA $_{3}^{\mathrm{Gly}}$ is very similar to that of the valine tRNA, tRNA ${ }_{2 \mathrm{~B}}^{\mathrm{Val}}$, which was analysed by Yaniv and Barrell. 78 per cent of the sequences of these two tRNAs are, in effect, identical and what few differences there are between the two molecules are to be found in their hydrogen bonded stems rather than their single-stranded loops. It seems therefore that the base sequences of these double-helical stems provide recognition signals which allow the glycyl and valyl tRNA synthetases to distinguish their respective tRNAs. Whether, however, this is a general rule, and the hydrogen bonded stems of all tRNAs provide recognition signals for their respective synthetases, remains very much an open question.

from the phase-diffraction grating, formed by the ultrasonic pulse train, gave diffraction maxima separated by only 66 arcsec from the undeflected light using sheer waves of frequency 2 $\mathrm{MHz}$. 